\title{
Superimposed Voltage Testing of HVDC Equipment with Oscillating Impulse Voltage
}

Hallas, Martin; Dorsch, Christian; Hinrichsen, Volker

(2018)

DOI (TUprints):

Lizenz:

Publikationstyp:

Fachbereich:

Quelle des Originals: https://doi.org/10.25534/tuprints-00014261

lediglich die vom Gesetz vorgesehenen Nutzungsrechte gemäß UrhG Konferenzveröffentlichung

18 Fachbereich Elektrotechnik und Informationstechnik https://tuprints.ulb.tu-darmstadt.de/14261 


\title{
Superimposed Voltage Testing of HVDC Equipment with Oscillating Impulse Voltage
}

\author{
M. Hallas, C. Dorsch, V. Hinrichsen \\ Technische Universität Darmstadt \\ Fraunhoferstraße 4, Darmstadt, Germany \\ hallas@hst.tu-darmstadt.de
}

\begin{abstract}
Superimposed voltage testing with oscillating impulse voltage offers many advantages for long-term testing of large test assemblies. The contribution will show how superimposed voltage testing with OLI/OSI voltages can be realized in high voltage laboratories and discuss superimposed OLI/OSI testing using spark gaps as coupling elements. Laboratory results have shown that with the help of spark gaps superposition of OLI/OSI voltage can be realized more easily than it is the case for conventional standard LI and SI voltage. Certain effects are observed at superimposed OLI/OSI testing. They influence amplitude and shape of the test voltage and thus have to be considered for generator design and during testing. Experimental laboratory results as well as simulations elaborate the effects.
\end{abstract}

\section{INTRODUCTION}

High voltage DC equipment is tested with superimposed voltages to simulate failures during operation. Cigré JWG D1/B3.57 "Dielectric Testing of gas-insulated HVDC systems" discusses prototype installation tests with a testing period of one year. As part of these tests, superimposed voltage tests shall be performed [1]. Prequalification tests of DC cables also demand superimposed voltage at the end of the test period of one year. For example, the DC cables shall have a length of approximately $100 \mathrm{~m}$ [2]. Common to both examples are the large test samples. Prototype installation tests of GIL (gas insulated line) assemblies would demand lengths similar to those of DC cables. Test procedures should be as close to practice as possible. Therefore, users of HVDC equipment would desire superimposed voltage testing of the total test object during the long-term tests. In practice, superimposed voltage testing on large test assemblies is difficult to realize because of travelling wave effects and the high capacitance of the test object. Assuming a length of $100 \mathrm{~m}$ for the test object and a propagation speed equal to the speed of light $\left(\mathrm{c}_{0}=300 \mathrm{~m} / \mu \mathrm{s}\right)$, the resulting travelling time of $0,33 \mu \mathrm{s}$ along the test object is in the range of the rise time of a standard LI voltage with a front time of $1.2 \mu \mathrm{s}$. In conclusion, overvoltages due to travelling wave effects occur along the test object, which results in unreasonable testing [3]. Furthermore, large test generators are required for a $100 \mathrm{~m}$ assembly. E.g. for DC cables lengths of only $30 \mathrm{~m}$ shall be tested due to laboratory limitations [2]. To overcome these problems, oscillating impulse voltage can be used (OLI/OSI - oscillating lightning/switching impulse). Cigré JWG D1/B3.57 also discusses to accept this type of superimposed impulse voltage for long-term tests [1]. As a major advantage of using OLI/OSI smaller generators can be used. Furthermore, longer front times are allowed [4]. Mobile OLI/OSI generators are typically used for on-site testing after the installation of cable- or gasinsulated-systems. The same procedure could be used during long-term testing. After or during long-term tests of HVDC equipment in the laboratory, suitable generators could be transported directly to the test object to perform superimposed voltage tests. The change of the front time of $1.2 \mu$ s for standard LI voltage to maximum $20 \mu$ s for OLI voltage will have effects on the dielectric response of the HVDC equipment. Studies on gas-insulated systems have shown a reduction of the dielectric performance by approximately $10 \%$ when using the maximum allowed front time of OLI voltage $[3 ; 5]$. Further studies on $\mathrm{SF}_{6-}$ insulated systems have not shown any differences between using OLI voltage and standard LI voltage as long as $T_{1}$ is equal. Based on this experience, the on-site test voltage level for OLI/OSI is set to $80 \%$ of the IEC test voltage level [6]. To avoid overstress during long-term tests of gas-insulated systems, a similar approach is reasonable [1]. In conclusion, using superimposed oscillating impulse voltage offers a cost-efficient solution for long-term testing of large DC test assemblies. Tests at only $80 \%$ of the IEC test level seem reasonable.

\section{SuPERIMPOSED Voltage TESTING}

Superimposed voltage testing requires coupling and blocking elements to superimpose the impulse voltage to the test sample and to block it from the DC voltage source. Coupling capacitors or spark gaps are common coupling elements [1]. Figure 1 shows the conventional test circuit for superimposed voltage testing [7]. Coupling capacitors offer the advantage of a reliable superposition during testing, because the impulse voltage is directly added to the DC voltage. To minimize the voltage drop across the coupling capacitor, a capacitance at least ten times higher than that of the test object is required. This results in expensive investments for the coupling capacitors. Superposition with spark gaps works by discharge of the spark gap, which directly applies the impulse voltage at the test sample. Spark gaps are cost efficient and available in every high voltage laboratory. Nevertheless, literature reports practical problems to achieve reliable discharge of the spark gap. In case of discharge, an influence on the impulse voltage waveform can be obtained. 


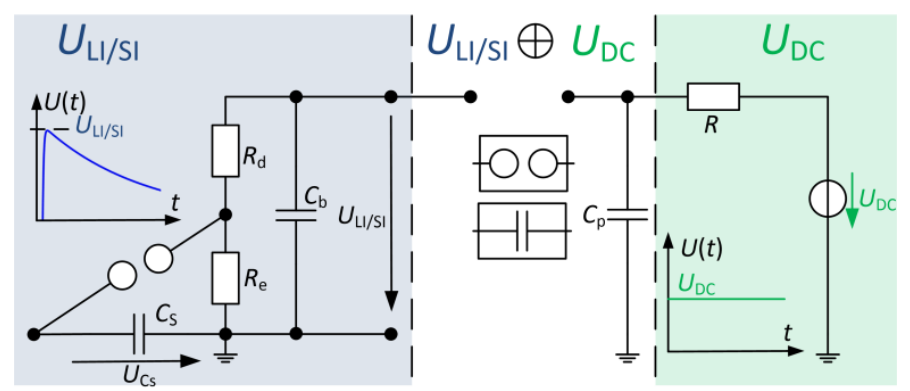

Figure 1. Test circuit for conventional superimposed voltage testing [7].

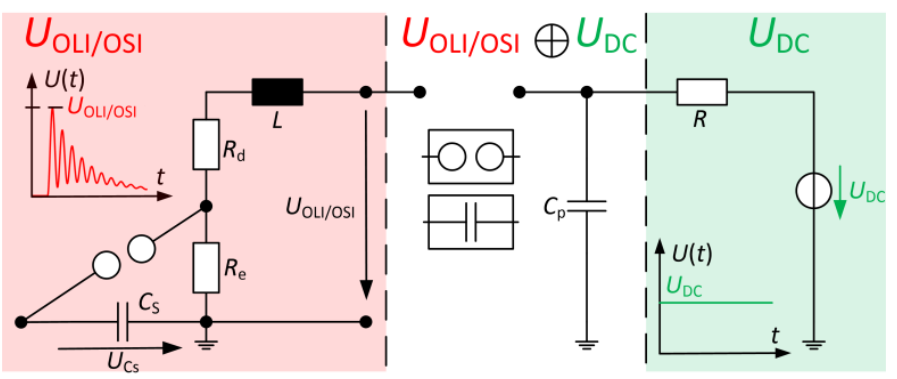

Figure 2. Test circuit for superimposed OLI/OSI testing.

Especially for SI voltage the spark gap may extinguish too early, which results in unrealistic testing. According to the literature, superimposed SI testing is generally more critical than LI testing [7].

So far, superimposed voltage tests have only been performed with conventional LI/SI voltages. Superimposed OLI/OSI voltage testing may be assumed to be similar, but no test experience is available so far. The circuit diagram for OLI/OSI voltage testing is shown in Figure 2. In comparison to Figure 1, the OLI/OSI generator does not have a load capacitor $C_{\mathrm{b}}$. The capacitance of the test object $C_{\mathrm{p}}$ directly serves as load capacitor. This means that in contrast to the standard LI/SI voltage test the load capacitance is fully charged to DC voltage before the impulse voltage is superimposed. This influences the superposition and results in certain special effects during the testing, which are described in chapter IV.

\section{SUPERPOSITION OF OSCILLATING VOLTAGE}

Superimposed OLI/OSI testing with coupling capacitor was performed in the laboratory for testing voltages with approximately $30 \mathrm{kV}$ DC and $60 \mathrm{kV}$ OSI. The test voltage levels with coupling capacitors could not be increased due to

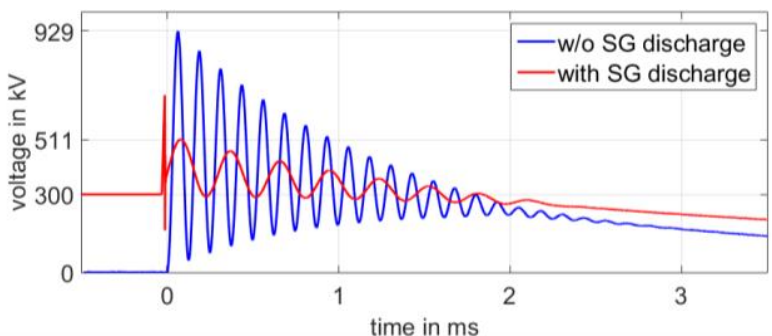

Figure 3. Voltage oscillograms with and w/o spark gap discharge ( $U_{\text {OLI/OSI }}$ Figure 2).
Table 1: Test parameters during OSI tests.

\begin{tabular}{|c|c|c|}
\hline & \multicolumn{2}{|c|}{ DC Assembly } \\
\hline Parameter & $300 \mathrm{kV}$ & $30 \mathrm{kV}$ \\
\hline Total impulse capacitance $C_{\mathrm{S}}$ & $15.6 \mathrm{nF}$ & $550 \mathrm{nF}$ \\
\hline Capacitance of test object $C_{\mathrm{p}}$ & $15.5 \mathrm{nF}$ & $138 \mathrm{nF}$ \\
\hline Total OSI inductance $L$ & $261 \mathrm{mH}$ & $42 \mathrm{mH}$ \\
\hline Front time resistor $R_{\mathrm{d}}$ & $220 \Omega$ & $5 \Omega$ \\
\hline Tail resistor $R_{\mathrm{e}}$ & $15 \mathrm{k} \Omega$ & $3.2 \mathrm{k} \Omega$ \\
\hline
\end{tabular}

laboratory limitations. A reliable superposition comparable to conventional testing could be observed.

Superimposed OLI/OSI voltage testing with spark gaps was performed in the laboratory up to $300 \mathrm{kV} \mathrm{DC}$. The investigation for $300 \mathrm{kV}$ DC voltage was performed for OSI voltage only, since literature describes SI voltage testing as more difficult to realize [7]. The focus of the investigation was on testing with spark gaps, due to the much lower costs compared to coupling capacitors. The test parameters are given in Table 1.

The capacitance of the test object during the $300 \mathrm{kV}$ tests was chosen to represent the capacitance of a typical $300 \mathrm{~m}$ GIL structure. Discharge of the spark gap could be performed very reliably for OLI/OSI voltage testing. Even tests at only $515 \mathrm{kV}$ OSI voltage, this menas $68 \%$ of $750 \mathrm{kV}$ (lowest IEC SI voltage test level for $300 \mathrm{kV}$ [8]), superimposed to $300 \mathrm{kV} \mathrm{DC}$ could be performed without problems. The gap distance of appr. $20 \mathrm{~cm}$, necessary at the least to isolate the $300 \mathrm{kV} \mathrm{DC}$ voltage, could even be increased while still achieving reliable spark gap discharge. Increasing the gap distance up to $50 \mathrm{~cm}$ and reducing the impulse voltage amplitude down to $420 \mathrm{kV}$ was possible.

Figure 3 shows oscillograms with and without discharge of the spark gap during superimposed OLI/OSI voltage testing The voltage with discharge of the spark gap (red curve) shows the expected behavior, which can be explained with the help of the circuit shown in Figure 2. When replacing the spark gap by a short-circuit (which represents the condition after an discharged spark gap), the conventional circuit for OLI/OSI voltage testing results, and therefore, the expected voltage shape will develop. Without discharge of the spark gap, the spark gap in Figure 2 remains as isolating gap and no superposition occurs. In conclusion, there is no load capacitor $C_{\mathrm{p}}$ in the circuit. Thus the impulse capacitor will discharge into the stray (earth) capacitances of the overall test setup. Impulse capacitor, impulse coil and earth capacitances form a resonant circuit of a much higher resonant frequency. And as the load capacitance is formed only by the small earth capacitances the generator's utilization factor $\eta=U_{\text {out }} / U_{\text {in }}$ reaches a value of around $2 \cdot C_{\mathrm{s}} /\left(C_{\mathrm{s}}+C_{\mathrm{b}}\right)$. In contrast, high load capacitances $C_{\mathrm{b}}$ would result in utilization factors close to those of conventional LI/SI voltage generators [4]. In conclusion, the voltage without discharge results in a very high and very steep voltage overshoot. The voltage overshoot of $U_{\mathrm{OLI} / O \mathrm{OSI}}$ in Figure 2 is the reason for the very reliable discharge behavior during superimposed testing.

Simulations with different load capacitance values show also voltage overshoots as long as the load capacitance is much 


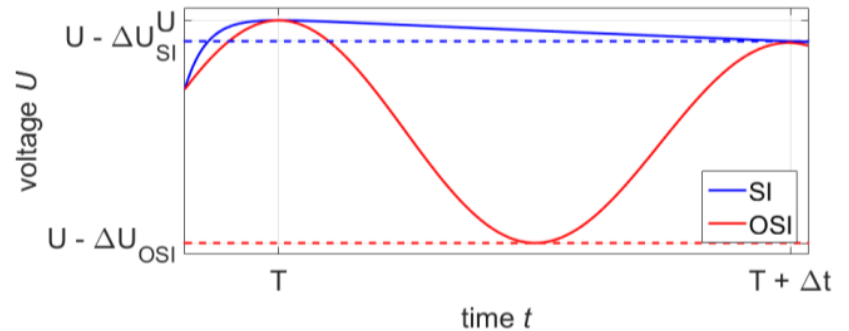

Figure 4. Comparison of the first peaks of OSI and SI voltage.

higher than the earth capacitance. Even for low load capacitance test arrangements, additional capacitors of some Nano-Farads in parallel to the test arrangement could be installed to provoke the effect in Figure 3 and achieve reliable discharge. In conclusion, reliable discharge is also possible for any other test arrangement.

During the investigations on superimposed OLI/OSI voltage testing, more than 1000 impulses with spark gaps were performed in the laboratory. The OLI/OSI voltage oscillograms did not show any indication of arc extinction in the spark gap, as it can be observed with superimposed standard LI/SI voltage testing. Furthermore, arc extinction in the spark gap due to zero crossing of the voltage could not be observed during OLI/OSI voltage testing. Figure 4 shows the differences between both voltage curves around the first voltage peak. The flat-top of SI voltage results in a low voltage difference $\Delta U_{\text {SI }}$ across the spark gap during the time $\Delta t$. This results in low arc energy and may therefore cause arc extinction during superimposed SI testing [7] OSI voltage does not have a flat-top, instead an oscillating current flows through the arc. The steady current flow in combination with the steady voltage difference $\Delta U_{\text {OSI }}$ across the gap during $\Delta t$ is thereby sufficient to "feed" the arc sufficiently, even in case of zero crossing of the OLI/OSI voltage.

\section{EFFECTS DURING SUPERIMPOSED OSI/OLI TESTING}

During superimposed OLI/OSI voltage testing, certain effects were observed. Since blocking capacitors directly add the impulse voltage to the DC voltage, the effects cause oscillograms different to those of Figure 5 and Figure 6. Nevertheless, the consequences are similar to those for testing with spark gaps. In both cases the DC voltage requires special consideration in order to achieve a suitable testing and generator design. The effects were observed during the test with DC voltages of some $30 \mathrm{kV}$, as well as during the tests at $300 \mathrm{kV}$. The following examples show the effects during testing with spark gaps.

The first effect influences the amplitude of the test voltage. Keeping the charging voltage of the impulse voltage generator constant and changing the DC voltage affects the amplitude of the resulting voltage. Figure 5 shows the effect during laboratory tests at $30 \mathrm{kV}$ DC voltage. The test parameters are given in Table 1. Compared to tests without any DC voltage, tests with same polarity of the DC voltage decrease the amplitude, while tests with opposite polarity of the DC voltage increase it. According to Figure 5, the amplitudes differ by $\pm 23 \%$. The effect gets less for $C_{\mathrm{s}} \approx C_{\mathrm{p}}$ and even tends to be inverted in case

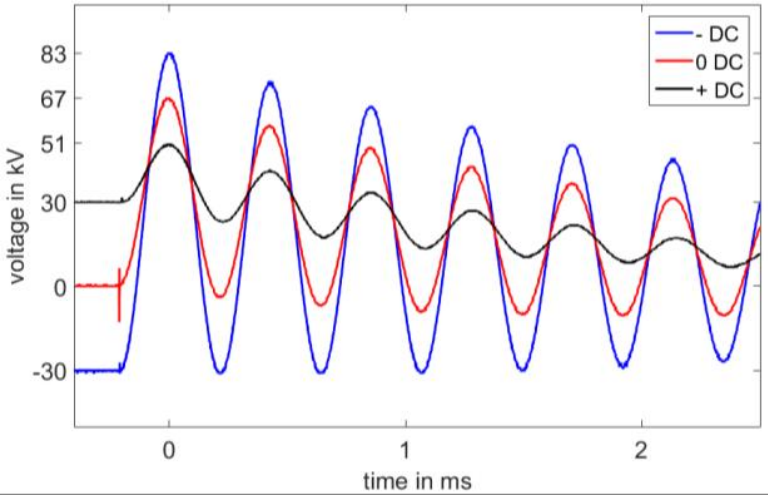

Figure 5: Change of amplitude during superimposed OLI/OSI testing with constant charging voltage.

of $C_{\mathrm{s}}<C_{\mathrm{p}}$. This means the amplitude tends to be reduced for opposite polarity and increased for same polarity. This inversion was observed especially during the $300 \mathrm{kV}$ DC tests. Simulations also point out this tendency. In conclusion, this effect has to be carefully considered in order not to overstress the test object. Adjusting the impulse voltage generator with zero DC voltage before superimposed testing with opposite polarity would result in a wrong amplitude of the superimposed testing. On the other hand, specifying the necessary OLI/OSI voltage generator for testing purposes without considering the DC voltage would result in too small test generators due to the amplitude reduction during superimposed tests.

The second effect influences the time parameters "front time" $\left(T_{\mathrm{p}}\right)$ and "time-to-half-value" $\left(T_{2}\right)$. Figure 6 shows this effect. The DC voltage influences the steepness of the voltage curve. A tangent drawn at the impulse voltage front intersects with the $\mathrm{x}$ axis with a variation in the range of $-10 \%$ to $+20 \%$ compared to the zero DC voltage level. The time-to-half-value also results in changes of $-32 \%$ to $+23 \%$ compared to the zero DC voltage case. Both of these time effects have to be considered during the design of the OLI/OSI generator for superimposed testing. The generator design might be suitable for testing without DC voltage, but due to the big changes of the time parameters, different values for impulse coil, front- or tail resistors may be required for superimposed testing.

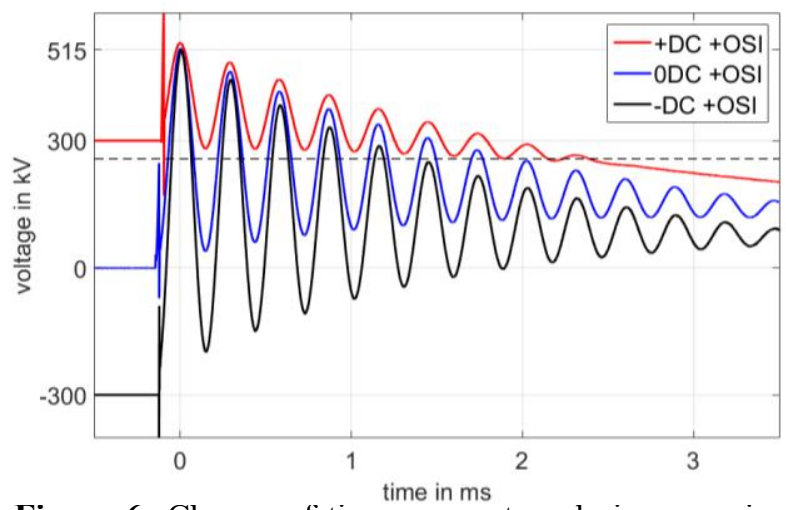

Figure 6: Change of time parameters during superimposed OLI/OSI voltage testing ( $U_{\text {OLI/OSI }}$ Figure 2). 
The effects shown in Figure 5 and Figure 6 can be explained with the electrical circuit in Figure 2. Both the impulse capacitor and the load capacitor are pre-charged with DC voltage. The capacitors as well as the coil and the resistors form a resonant circuit. With the same DC voltage at both capacitors, no oscillation will occur. But DC voltages of opposite polarity at both capacitors would result in a severe oscillation, because the voltages of both capacitors tend to equalize each other. This results in the change of amplitude in Figure 5 and the change of the time parameters in Figure 6, respectively.

Simulations also prove the plausibility of the observations made in the laboratory. Simulations for superimposed OLI/OSI voltage testing are performed mostly like those for conventional $\mathrm{LI} / \mathrm{SI}$ voltage testing. Major difference is the DC pre-stress at the test object $C_{\mathrm{p}}$ in Figure 2 at the beginning of the impulse. Simulations show the same effects as the laboratory results shown in Figure 5 and Figure 6. Figure 7 shows a comparison between simulations and laboratory results at $\pm 300 \mathrm{kV}$ DC. Frequency and front time of the voltage shapes fit very well. The amplitude of the further oscillation shows an increasing difference. The deviation between both curves can be explained with the voltage dependent arc-resistance. Following the voltage shape in Figure 7, the mean voltage level is decreasing, and thus the arc resistance increases. This also results in an increasing deviation with time and explains the different deviations in the $+300 \mathrm{kV}$ and $-300 \mathrm{kV}$ curves in Figure 7 . The $+300 \mathrm{kV}$ voltage shape has a much lower voltage difference in the beginning and, therefore, a much higher arc resistance. The higher arc resistance results in a higher difference between simulation and laboratory results as arc resistance has not been considered in the simulation. Furthermore, the deviation is smaller for tests at only $30 \mathrm{kV}$. Lower arc resistances occur due to the smaller gap distances, which also indicates the voltage dependent arc resistance. Statistical deviations of time parameters due to the arc resistance were not observed. Evaluating the differences between simulations and laboratory results shows a change of the time-to-half-value of approximately $15-20 \%$ due to the arc resistance. This also has to be considered in case of superimposed OLI/OSI voltage testing with spark gaps.

\section{CONCLUSION}

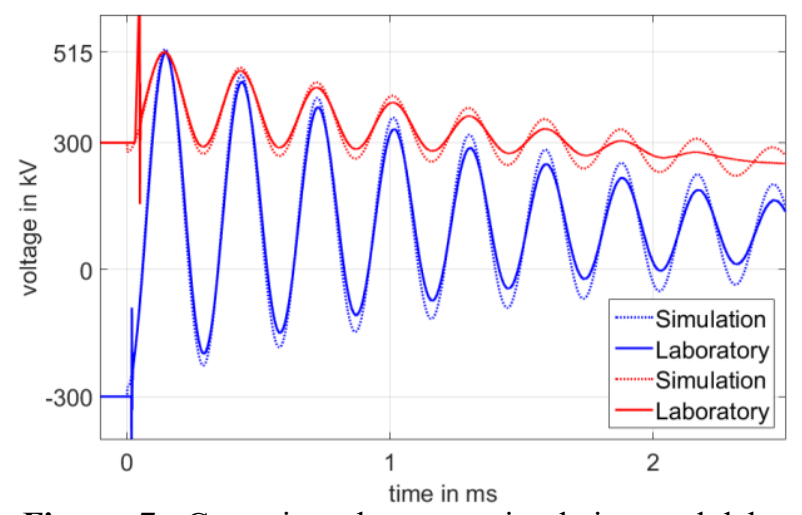

Figure 7: Comprison between simulation and laboratory results ( $U_{\mathrm{OLI} / \mathrm{OSI}}$ Figure 2).
Testing with OLI/OSI voltage is a cost-efficient means of performing superimposed tests on large test assemblies. Especially superimposed tests during long-term tests on DC cable or GIL assemblies with lengths of $100 \mathrm{~m}$ and more can be realized. Users of HVDC equipment would benefit from the more realistic test scenario. Superimposed voltage testing with OLI/OSI voltage is basically possible with coupling capacitors as well as with spark gaps. Testing with only $80 \%$ of the IEC impulse voltage test level is also possible with both coupling elements. Testing with spark gaps has no limitation due to unintended arc extinction or other effects well known for conventional impulse testing. During the design of an OLI/OSI voltage generator for superimposed testing, certain effects have to be considered. Compared to zero DC voltage, amplitude and time parameters of the impulses change depending on the DC voltage amplitude. Therefore, adjustment of the OLI/OSI voltage generator requires a careful approach. Furthermore, the influence of the arc resistance has to be considered for superimposed testing with spark gaps.

\section{ACKNOWLEDGMENT}

The authors gratefully acknowledge support of this work by the IWB-EFRE-Program by the State of Hessen (Funding Code 20002558).

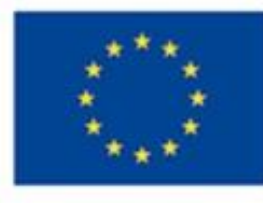

\section{EUROPEAN UNION European Regional Development Fund Investing in your future}

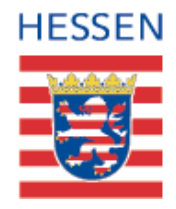

\section{REFERENCES}

[1] C. Neumann et al, "Some thoughts regarding prototype installation tests of gas-insulated HVDC systems," Cigré International Colloquium \& Exhibition, Winniepeg, 2017

[2] IEC 62895, "Cables with extruded insulation and their accessories for rated voltages up to $320 \mathrm{kV}$ for land applications," 2017

[3] U. Schichler, A. Diessner and J. Gorablenkow, "Dielectric on-site testing of GIL," Proceedings of the 7th International Conference on Properties and Applications of Dielectric Materials (Cat. No.03CH37417), 2003, pp. $15-18$ vol.1.

[4] E. Gockenbach and J. Meppelink, "Breakdown behaviour of nonuniform arrangements in air and SF6 using oscillating lightning impulse voltage," 1982 IEEE International Conference on Electrical Insulation, Philadelphia, PA, USA, 1982, pp. 206-210.

[5] W. Boeck et.al, "Insulating behaviour of SF6 with and without solid insulation in case of fast transients," Cigre Session Paris, report 15-07, 1986

[6] IEC 60060-3, "High voltage test techniques - Part 3: Definitions and requirements for on-site testing," 2006

[7] A. Voß, M. Gamlin, "Superimposed impulse voltage testing on extruded DC-cables according to IEC CDV 62895," ISH Buenos Aires, 2017

[8] IEC 60071-1: "Insulation co-ordination - Part 1: Definitions, principles and rules," 2006 